\title{
FORUM
}

\section{Berlin blassblau: zur Europapolitik der neuen Bundesregierung}

\author{
Joachim Wuermeling*
}

\begin{abstract}
„Pantone Reflex Blue“ - so lautet die technische Bezeichnung der Hintergrundfarbe der Europaflagge. Das kräftige Azurblau steht für ein starkes Europa. Eher blassblau hingegen startet die neue Bundesregierung ihre europapolitische Aktivität. Kein Wunder: Weder im Wahlkampf noch in den Koalitionsverhandlungen noch in der Debatte um das Regierungsprogramm haben Europafragen eine Rolle gespielt.

Das erstaunt, wird doch in Brüssel und Straßburg mit dem Lissabon-Vertrag, ein wahrhaft neues Kapitel der Europapolitik aufgeschlagen. Nach den jahrelangen, quälenden institutionellen Diskussionen und dem vorläufigen Abschluss der Aufnahme weiterer Staaten kann sich Europa mit voller Kraft endlich den Sachthemen zuwenden. An großen und drängenden Aufgaben mangelt es dabei nicht: Wirtschafts- und Finanzkrise, Klimawandel, demografische Lücke, Globalisierung der Politik.

Im folgenden Beitrag werden in einer Bestandsaufnahme die wesentlichen inhaltlichen, institutionellen und personellen Festlegungen für die neue Legislatur referiert und vor dem Hintergrund der Herausforderungen für die deutsche Europapolitik bewertet, bevor einige Anregungen für das Regierungshandeln gegeben werden.
\end{abstract}

\section{Neustart der Europapolitik: alles beim Alten?}

Inhaltlich und institutionell setzt die neue Bundesregierung auf Kontinuität. Dabei haben die Begleitgesetze zur Lissabon-Ratifizierung, der Lissabon-Vertrag selbst und das Urteil des Bundesverfassungsgerichts die Spielregeln für ihr Handeln grundlegend verändert.

Auf gerade einmal vier der 132 Seiten des Koalitionsvertrags werden die Grundsätze der Europapolitik skizziert. Abgesehen von der Passage über den Türkei-Beitritt sollen die Verhandlungen in der entsprechenden Arbeitsgruppe, die zugleich die Themen Außen-, Sicherheits- und Entwicklungspolitik behandelte, konsensual verlaufen sein. Das war vielleicht auch dem Umstand geschuldet, dass - anders als bei früheren Koalitionsbildungen - die Parteien Bundes- und Landes- aber keinen einzigen Europapolitiker in die Delegationen aufgenommen hatten.

Inhaltlich sind die Festlegungen sehr allgemein gehalten: Es findet sich das Bekenntnis zu einer ,leistungsfähige[n] und selbstbewusste[n] EU“, die demokratisch, transparent, bürgernah und nach freiheitlichen Grundsätzen gestaltet sein soll. Europa soll den Rahmen abgeben für ein eigenverantwortliches und solidarisches Leben. Demgegenüber soll sich die Europäische Union auf das Wesentliche beschränken. Bei der Umsetzung europäischer Richtlinien wird der deutsche Gesetzgeber über die EU-Vorgaben nicht hinausgehen. Besonderen Wert legen die Koalitionäre auf die Einhaltung der Prinzipien der begrenzten Einzelermächtigung, der Subsidiarität und der Verhältnismäßigkeit. Grenzüberschreitende EU-

* Dr. jur. Joachim Wuermeling, LL.M., Sprecher von Europa-Professionell, Staatssekretär a. D., MdEP a. D., Mitglied der Hauptgeschäftsführung des Gesamtverbandes der Deutschen Versicherungswirtschaft, Berlin. 
Sozialsysteme werden abgelehnt. Ausdrücklich unterstrichen werden die Ziele der Wettbewerbsfähigkeit und des Wirtschaftswachstums.

Neben diesem betont freiheitlichen und insbesondere wirtschaftsliberalen Einschlag lassen noch die Ausführungen zu den EU-Finanzen aufhorchen. Denn es werden substanzielle Vorschläge für eine ,Umschichtung ‘ im EU-Haushalt angekündigt. Mehr Mittel sollen für die Gemeinsame Außen- und Sicherheitspolitik, die europäische Infrastruktur und die Forschung eingesetzt werden. Da aber eine Erhöhung der Beitragszahlungen abgelehnt wird, muss dies wohl mit Kürzungen an anderer Stelle, etwa bei den Agrarausgaben oder der Regionalpolitik ermöglicht werden.

Die europapolitischen Aussagen sind allerdings nicht auf das EU-Kapitel beschränkt. Überraschend häufig, an 40 weiteren Stellen, werden im Rahmen der Fachpolitiken europapolitische Ziele festgelegt, von der vollständigen Öffnung der Eisenbahnmärkte über die Stärkung der Versicherungswirtschaft bei der Ausgestaltung der neuen Solvenzregeln bis hin zur Harmonisierung der Normen für Rüstungsexporte.

An der Organisation der Europapolitik in der Bundesregierung ändert sich nichts. Die Schaffung eines deutschen Europaministers im Kanzleramt, wie sie seit Jahren von fast allen Seiten für erforderlich gehalten wird, scheiterte erneut an dem Machtanspruch des Bundesaußenministers und damit des kleineren Koalitionspartners. So wird auch künftig die Koordinierungsfunktion zwischen dem Bundeskanzleramt, dem Auswärtigen Amt (insbesondere für Außenpolitik, Inneres und Justiz, Finanzen) und dem Bundeswirtschaftsministerium (Wirtschaft, Umwelt, Soziales) aufgeteilt. Den Vorsitz bei den Europastaatssekretären der Bundesregierung teilen sich weiterhin das Auswärtige Amt und das Bundeswirtschaftsministerium. Politisch verantwortlich werden im Bundeskanzleramt Ronald Pofalla als Kanzleramtsminister, im Auswärtigen Amt Werner Hoyer als Staatsminister und im Bundeswirtschaftsministerium Peter Hintze als Parlamentarischer Staatssekretär sein.

Als Ergebnis der Koalitionsverhandlungen benannte die neue Bundesregierung überraschend den baden-württembergischen Ministerpräsidenten Günther Oettinger für das Amt des deutschen EU-Kommissars. Dem Vernehmen nach hatten zuvor eine Reihe anderer Politiker eine Nominierung abgelehnt.

Im neu gewählten Deutschen Bundestag wird es wieder einen Ausschuss für die Angelegenheiten der Europäischen Union geben, dem erneut der CDU-Abgeordnete Gunther Krichbaum vorsteht. Als europapolitische Sprecher der Fraktionen wurden Michael Stübgen (CDU/CSU), Axel Schäfer (SPD), Michael Link (FDP), Manuel Sarrazin (Die Grünen) und Diether Dehm (Die Linke) gewählt. Übrigens wurden in diesen Monaten auch die Vertretungen der Europäischen Kommission und des Europäischen Parlaments in Berlin mit Matthias Petschke und Frank Piplat neu besetzt.

Mit dem Lissabon-Vertrag und den Begleitentscheidungen werden sich die rechtlichen Grundlagen und in der Folge die politischen Bedingungen für die deutsche Europapolitik ganz erheblich ändern: Der Lissabon-Vertrag selbst bezieht die nationale Politik stark in den europäischen Prozess ein. Die nationalen Parlamente werden an dem europäischen Gesetzgebungsprozess beteiligt und erhalten mit dem Subsidiaritätseinspruch ein formelles Instrument. Mit einem europäischen Bürgerbegehren können auch die Wähler selbst Themen für Brüssel setzen.

Die Begleitgesetze zum Lissabon-Vertrag stärken deutlich die Mitwirkungs- und Informationsrechte des Bundestags und des Bundesrats. Das europapolitische Handeln der Bundesregierung dürfte in Zukunft maßgeblich von der Haltung des Bundestages bestimmt werden. Anders als bei nationalen Themen, in denen die Fragen zumeist in der Regierungskoalition vorbesprochen wurden, kann es bei der offenen und kritischen Meinungsbildung 
unter den Abgeordneten zu europäischen Fragen zu Konflikten mit der Regierung auch kommen, wenn die Mehrheitsverhältnisse eigentlich festliegen.

Dazu wird der Bundestag auch durch das Lissabon-Urteil des Bundesverfassungsgerichts veranlasst, das dem deutschen Parlament eine starke ,Integrationsverantwortung ' ins Stammbuch geschrieben hat. Es sieht die demokratische Legitimation europäischer Entscheidungen in erster Linie durch die nationalen Parlamente vermittelt.

Bemerkenswert erscheint in diesem Zusammenhang, dass sich in den letzten Jahren in Berlin schon eine sehr dynamische europapolitische Szene entwickelt hat. In Bundesministerien, Landesvertretungen, Verbänden, Lobby und Medien ist eine breite europapolitische Expertise entstanden, sei es durch die Befassung mit den europäischen Themen oder durch Mitarbeiter, die zuvor in Brüssel gearbeitet haben. Ein vielfältiges Angebot an europapolitischen Veranstaltungen führt zu einem regen Austausch. Netzwerke und Think Tanks wie die Europäische Bewegung, die Hauptstadtgruppe der Europa-Union Deutschland Europa-Professionell, die politischen Stiftungen oder das Institut für Europäische Politik bilden Foren und Plattformen für die Formulierung von Europapolitik in Berlin.

\section{Deutschland in Europa: ,weiter so' keine Alternative}

Das platte Diktum , weiter so ist keine Alternative‘ darf heute einmal für die deutsche Europapolitik fruchtbar gemacht werden. Denn der eher verhaltene und defensive europapolitische Aufschlag von ,Merkel II ' hat den einen oder anderen überzeugten Europäer enttäuscht.

Welche praktische Politik aber daraus entsteht, ist damit keineswegs ausgemacht. Bundeskanzlerin Angela Merkel hat in der letzten Legislaturperiode unter ähnlichen Ausgangsbedingungen als Europapolitikerin überzeugt und sich in der deutschen Ratspräsidentschaft 2007 großes Ansehen in Brüssel und Straßburg erworben. Aus der unprätentiösen Herangehensweise, die auch den alten Koalitionsvertrag kennzeichnete, sind richtungweisende Impulse und bahnbrechende Entscheidungen entstanden, etwa der Lissabon-Vertrag, die europäische Energie- und Klimapolitik sowie das einheitliche Auftreten in internationalen Verhandlungen.

Deshalb ist es jetzt wichtig, dem sich ausbreitenden Eindruck von einem retardierenden Desinteresse Deutschlands an Europa kraftvoll entgegenzutreten. Sätze wie: „But just do not look to Germany for fresh ideas"1 oder „Der thematische Kreis, über den in Deutschland diskutiert wird, hat sich verengt“2 oder „Deutlicher könnte Merkel kaum signalisieren, dass ihr Europa schnurz egal ist" ${ }^{* 3}$ sollten nicht mehr zu lesen sein. Die Bundeskanzlerin selbst hat in ihrer ,Humboldt-Rede‘ am 27. Mai 2009 betont, dass das Eintreten für deutsche Interessen und der „Blick für das Ganze [...] zwei Seiten derselben Medaille“ sind.

Der Lissabon-Vertrag wird zu einem Integrationsschub und zu einer „,so nie erlebten Europäisierung der deutschen Politik ${ }^{\star 4}$ führen. Der Methode Monnet und der institutionellen Methode wird eine ,politische Integrationsmethode“ folgen. ${ }^{5}$ Wie ein „Europa der Ergebnisse" sich in mannigfachen konkreten Vorhaben niederschlagen wird, hat Kommissionspräsident José Manuel Barroso in seinen ,politischen Leitlinien ‘ bereits dokumentiert. ${ }^{6}$

\footnotetext{
Gideon Rachman: Germany retreats to old certainties, in: FT.com, 21.09.2009.

Peter Sloterdijk, in: Süddeutsche Zeitung, 10.11.2009.

Welt am Sonntag: Deutsche Politiker sind die größten Euroskeptiker, 08.11.2009.

Joachim Fritz-Vannahme: Mehr Europa wagen, Bertelsmann Stiftung: spotlight europe 2009/08.

Näher dazu Joachim Wuermeling: Vom Verfassungsentwurf zum Reformvertrag: Auf dem Weg zur politischen Integrationsmethode?, in: Ulrich Fastenrath/Carsten Nowak (Hrsg.): Der Lissabonner Reformvertrag. Änderungsimpulse in einzelnen Rechts- und Politikbereichen, Berlin 2009, S. 33-46, hier S. 39 ff.

6 José Manuel Barroso: Politische Leitlinien für die nächste Kommission, Brüssel, 30.09.2009.
} 
Dabei muss auch dieser Bundesregierung klar sein, dass ihr eigener politischer Erfolg von der Gestaltung der europäischen Rahmenbedingungen abhängt. Ohne Wirtschaftswachstum im europäischen Binnenmarkt wird es keinen deutschen Aufschwung geben, ohne eine europäische Finanzaufsicht keine Finanzmarktstabilität, ohne europäische Energiepolitik keine sicherere Versorgung, ohne europäische Maßnahmen zum Klimawandel kein Aufhalten der Erderwärmung, ohne europäische Wettbewerbspolitik keine fairen Bedingungen für Unternehmen, ohne europäische Verbrechensbekämpfung keine innere Sicherheit, ohne europäische Forschungskooperation kein technischer Vorsprung, um nur einige Beispiele zu nennen.

Von Deutschland als größtem Mitgliedstaat in Europas Mitte wird von den Partnern Integrationskraft, Gestaltungswille und politische Führung erwartet. Dabei geht es weitaus weniger um die Entfaltung von deutschem Einfluss als um die Wahrnehmung von Verantwortung für Europa. Es geht weniger um einen pauschalen Integrationsschub als um die ausstehende Lösung von Problemen, die nationale Politik nicht mehr alleine bewältigen kann. Es geht weniger um die immer weitere Einebnung der unterschiedlichen Lebensverhältnisse oder gar der wohlmeinenden Gängelung des Bürgers als um das Offenhalten der Freiräume durch das Wegräumen von Hindernissen. Und schließlich geht es nicht um die Perfektionierung der nach innen gerichteten Politiken, sondern um die Behauptung Europas in dem entstehenden globalen Politikraum.

Nachdem sich „,bei der Suche nach demokratischer Teilhabe in Europa der Fokus auf die nationale Ebene" 7 verschoben hat, muss die neue Bundesregierung ihren Beitrag leisten zur Entwicklung Berlins als einem Ort, an dem Europapolitik gemacht wird. Die europapolitische Arbeit darf sich nicht darauf beschränken, von Berlin aus europapolitische Prozesse in Brüssel und Straßburg zu beeinflussen. Berlin muss selbst zum ,europäischen Politikplatz werden. Die Hauptstadt darf nicht nur Adressat von Informationen sein. Hier müssen politische Diskussionen stattfinden, die zu europapolitischen Entscheidungen führen. Die ,innenpolitische Dimension der Europapolitik [muss] stärker im einheimischen parlamentarischen Diskurs “8 abgehandelt werden. Dabei müssen die parlamentarischen Stränge genauso aktiviert werden wie die Kommunikationswege zwischen Ministerien und Verbänden, Wissenschaft und Politik sowie Medien und Öffentlichkeit.

\section{Sieben Anregungen für einen Neustart}

Damit eine neue Phase der Europapolitik in Berlin eingeläutet werden kann, bedarf es einiger Anstrengungen. Dazu gibt es viele Ideen. Ich möchte an dieser Stelle Bezug nehmen auf sieben Empfehlungen, die Europa-Professionell, ${ }^{9}$ die Hauptstadtgruppe der EuropaUnion Deutschland, zu Beginn der neuen Legislaturperiode vorgelegt hat. Die Empfehlungen, an denen ich selbst mitgewirkt habe, verfolgen drei Ziele: den Ausbau der Führungsund Integrationsrolle Deutschlands, die Stärkung des deutschen Einflusses auf europäische Entscheidungen und die Entwicklung eines echten Europapolitikplatzes Berlin. Die Empfehlungen lauten im Einzelnen:

7 Timm Beichelt/Anja Thomas: National politics matter! - Die Suche nach einer europäischen Verfassung und die Stärkung der nationalen Legitimationsgrundlagen der EU, in: integration 3/2009, S. 282-294, hier S. 294.

8 Peter-Christian Müller-Graff: Europa kann mit dem Lissabon-Urteil leben, in: EurActiv.de, 24.09.2009.

9 Europa-Professionell ist die Hauptstadtgruppe der Europa-Union Deutschland. Mitglieder sind in Berlin beruflich mit europapolitischen Fragen befasste Experten aus Politik, Verwaltung, Verbänden und Unternehmen. Europa-Professionell zählt derzeit etwa 150 Mitglieder. 
Die neue Bundesregierung sollte eine detaillierte deutsche, Europa-Agenda' für die kommende Legislaturperiode aufstellen.

Deutschland mangelt es in der Europäischen Union an europapolitischem Profil. In Brüssel wird nicht hinreichend deutlich, wofür Deutschland in Europa steht, was es in der Europäischen Union eigentlich erreichen will. Zwar werden zu einzelnen Dossiers die Positionen deutlich dargelegt. Was Deutschland von Europa insgesamt erwartet, ist aber nicht konkret genug zu greifen. Dabei hätte Deutschland gerade in dieser Phase der europäischen Integration die Chance, Ideengeber, Gestalter und Mittler zu sein, um der Europäischen Union neue Schlagkraft zu geben.

Durch die Erarbeitung einer entsprechenden Agenda sollte sich die Bundesregierung zunächst selbst darüber klar werden, welche Ziele sie in Europa in den nächsten vier Jahren verfolgt. Damit wird zugleich eine strategische und konzeptionelle Verantwortung für Europa insgesamt übernommen. Schließlich führt die Erarbeitung einer solchen Agenda zu fruchtbaren europapolitischen Diskussionen innerhalb Deutschlands.

Die neue Bundesregierung sollte sich rascher positionieren und ihre Haltung im Laufe des Entscheidungsprozesses flexibler anpassen.

Entscheidungsprozesse in Brüssel laufen nach ihrem eigenen Rhythmus ab und nehmen keine Rücksicht auf den Zeitbedarf der Meinungsbildung in Berlin. Der Einfluss der Bundesregierung hängt davon ab, dass frühzeitig die eigene Position eingebracht wird. Im Laufe der Beratungen ergeben sich ständig neue Verhandlungssituationen, auf die flexibel reagiert werden muss.

Die neue Bundesregierung sollte kontinuierliche Präsenz gegenüber allen europäischen Institutionen und auf allen Ebenen zeigen.

Die Zahl der relevanten Entscheidungsträger in EU-Kommission, Europäischem Parlament und den nationalen Regierungen dürfte bei mehreren 10.000 liegen. Deshalb ist es erforderlich, in den eigenen Fachzirkeln kontinuierlich Netzwerke zu bilden und informelle Kontakte zu pflegen. Für diese systematische Europaarbeit muss die notwendige Zeit zur Verfügung stehen. Das Engagement auch auf Beamtenebene muss gewürdigt werden.

Durch eine kontinuierliche Präsenz auf allen Ebenen und in allen Institutionen sowie eine breit angelegte Bildung von Netzwerken sollte die Bundesregierung die Grundlage dafür legen, dass in spezifischen Verhandlungs- und Konfliktsituationen auf einer guten Grundlage agiert werden kann. Die Bundesregierung muss durch ein verstärktes Monitoring insbesondere der Arbeit der Europäischen Kommission und des Europäischen Parlaments jederzeit ein genaues Bild von der Lage in Brüssel haben. Die handelnden Vertreter der Bundesregierung sollten in der jeweiligen Brüsseler, Szene ‘ ein Begriff sein, auch wenn sie ihren Dienstsitz in Berlin haben.

Die neue Bundesregierung sollte ihr europapolitisches Handeln gegenüber der Öffentlichkeit, den Parlamenten und den beteiligten Kreisen transparent machen.

Das Regierungshandeln in Brüssel und die Festlegung von europapolitischen Positionen der Bundesregierung in Berlin sind derzeit praktisch nicht Gegenstand der politischen Debatte. So wird in Berlin eine große Distanz zu den Brüsseler Vorgängen empfunden. Mangels früherer Informationen wird die allgemeine Öffentlichkeit in Deutschland immer wieder von Entscheidungen der Europäischen Union, überrascht', die in Brüssel ein ,alter Hut' sind. Die ,Integrationsverantwortung" wird weitgehend hinter verschlossenen Türen 
wahrgenommen. EU-Angelegenheiten werden nicht als politisch, sondern als routinemäßige Facharbeit wahrgenommen.

Für europapolitische Vorgänge muss in Deutschland die Öffentlichkeit hergestellt werden. Die Positionierung der Bundesregierung, das Handeln im Ministerrat und der Fortgang der Beratungen sollte von einer Debatte begleitet werden, so, wie das bei nationalen Vorhaben auch der Fall ist.

Die neue Bundesregierung sollte effizientere und klarere Entscheidungsstrukturen etablieren.

Koordinierende Funktionen innerhalb der Bundesregierung haben gegenwärtig das Bundeskanzleramt, das Auswärtige Amt und das Bundeswirtschaftsministerium inne. Die Federführung für die einzelnen Dossiers liegt bei den Fachressorts. Bundestag und Bundesrat werden in Zukunft stärker einzubeziehen sein. Diese komplizierten und fragmentierten Strukturen haben zur Folge, dass dem Handeln der Bundesregierung ein komplexer interministerieller und innerstaatlicher Beratungsprozess vorausgeht. Nach dem Inkrafttreten des Lissabon-Vertrags muss die Bundesregierung einen politischen Verantwortlichen für den Rat für Allgemeine Angelegenheiten benennen.

Die erforderliche Koordinierung soll einerseits effizient genug sein, um auch kurzfristig reagieren zu können, andererseits eine breit angelegte Meinungsbildung ermöglichen. In den Verfahren muss auch politisch und darf nicht nur fachlich entschieden werden.

Die neue Bundesregierung sollte die europäische Personalpolitik strategischer, vorausschauender und nachhaltiger gestalten.

Deutschland ist im Personal der europäischen Institutionen nach wie vor unterrepräsentiert. Da die Funktionen formell nicht nach Proporz und zum Teil gar nicht von außen besetzt werden können, kann dies nicht von heute auf morgen geändert werden. Personalpolitik in Europa muss langfristig angelegt werden.

Mit einer systematischen, strategischen und mittel- und langfristig angelegten Personalpolitik sollte die Präsenz Deutschlands in den europäischen Institutionen verbessert werden. Der Stellenwert der EU-Arbeit in Brüssel, aber auch in den Ministerien in Berlin muss höher geschätzt werden.

Die neue Bundesregierung sollte mit einer integrierten, modernen und adressatengerechten Kommunikation die Öffentlichkeit über die europapolitischen Vorgänge aus deutscher Sicht informieren.

Die deutsche Öffentlichkeit nimmt europapolitische Vorgänge nur punktuell wahr. Debatten entzünden sich weniger an den Tagesereignissen der europapolitischen Agenda, sondern an ,Aufregerthemen', die zufällig, punktuell und selektiv in den öffentlichen Fokus gelangen. So nimmt das deutsche Publikum nur bedingt teil an dem Mainstream der politischen Debatte in Brüssel und Straßburg. Europäische Öffentlichkeit in Brüssel und deutsche Öffentlichkeit haben nicht den gleichen Schritt und nur eine geringe Teilmenge. Das trägt zur Entfremdung der deutschen Bevölkerung von Europa wesentlich bei.

Die Öffentlichkeitsarbeit sollte dem europapolitisch potenziell interessierten Bürger einen sachlich fundierten Einblick in die europapolitischen Sachverhalte aus deutscher Sicht ermöglichen. 
Fazit

Stärken und Erfolge der deutschen Europapolitik der vergangenen Jahre und Jahrzehnte sind unbestreitbar. Dennoch stellen die politischen, institutionellen und praktischen Veränderungen auf deutscher und europäischer Ebene neue und höhere Anforderungen. Die Bundesregierung sollte die neue Legislaturperiode zum Anlass für einen Neustart ihrer europapolitischen Arbeit nehmen. Sie kann dabei auf einer guten Infrastruktur und breiten Erfahrungen aufbauen. Eine nüchterne Analyse der Stärken und Schwächen wird dabei schnell den Reformbedarf zutage fördern.

\section{Erstes Nachschlagewerk für deutsch-französische Beziehungen}

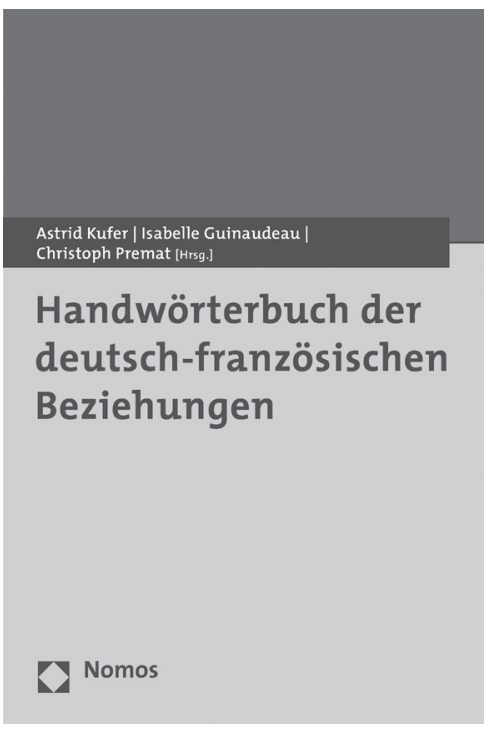

Bitte bestellen Sie im Buchhandel oder versandkostenfrei unter $>$ www.nomos-shop.de

\author{
Handwörterbuch der deutsch- \\ französischen Beziehungen \\ Herausgegeben von Astrid Kufer, \\ Isabelle Guinaudeau und \\ Christophe Premat \\ 2009, 245 S., brosch., 24,- $€$, \\ ISBN 978-3-8329-4807-8
}

Mit diesem Handwörterbuch liegt erstmals ein umfassendes Nachschlagewerk zu den deutsch-französischen Beziehungen vor. In über 90 Beiträgen stellen Nachwuchswissenschaftler aus verschiedenen Disziplinen die wichtigsten Institutionen, Akteure und Mittlerfiguren aus Politik, Gesellschaft und Kultur sowie historische Wendepunkte der deutsch-französischen Beziehungen vor. 